\title{
Online chromatic dispersion monitoring and compensation using a single inband subcarrier tone
}

Petersen, Martin Nordal; Pan, Z.; Lee, S.; Havstad, S. A.; Willner, A. E.

Published in:

I E E E Photonics Technology Letters

Link to article, DOI:

$10.1109 / 68.992614$

Publication date:

2002

Document Version

Publisher's PDF, also known as Version of record

Link back to DTU Orbit

Citation (APA):

Petersen, M. N., Pan, Z., Lee, S., Havstad, S. A., \& Willner, A. E. (2002). Online chromatic dispersion monitoring and compensation using a single inband subcarrier tone. I E E E Photonics Technology Letters, 14(4), 570-572. https://doi.org/10.1109/68.992614

\section{General rights}

Copyright and moral rights for the publications made accessible in the public portal are retained by the authors and/or other copyright owners and it is a condition of accessing publications that users recognise and abide by the legal requirements associated with these rights.

- Users may download and print one copy of any publication from the public portal for the purpose of private study or research.

- You may not further distribute the material or use it for any profit-making activity or commercial gain

- You may freely distribute the URL identifying the publication in the public portal 


\title{
Online Chromatic Dispersion Monitoring and Compensation Using a Single Inband Subcarrier Tone
}

\author{
M. N. Petersen, Z. Pan, S. Lee, S. A. Havstad, Member, IEEE, and A. E. Willner, Senior Member, IEEE
}

\begin{abstract}
In this letter, we demonstrate a simple technique for dispersion monitoring by adding a single inband subcarrier tone to the transmitted data signal. A measurable dispersion of up to 1200 $\mathrm{ps} / \mathrm{nm}$ is demonstrated in a 10-Gb/s channel using a 7-9 GHz subcarrier and the addition of the subcarrier induced a power penalty of $<0.5 \mathrm{~dB}$. Dynamic ranges exceeding $20 \mathrm{~dB}$ and resolution sensitivities better than $10(\mathrm{ps} / \mathrm{nm}) / \mathrm{dB}$ are shown. With an 8-GHz tone and a $15 \%$ modulation depth for $10-\mathrm{Gb} / \mathrm{s}$ signals, we show a measurement range of $975 \mathrm{ps} / \mathrm{nm}$ with a 22-dB dynamic range. We used the monitor output signal to achieve accurate tunable dispersion compensation.
\end{abstract}

Index Terms-Chromatic dispersion, dispersion compensation, dispersion monitoring.

\section{INTRODUCTION}

$\mathbf{F}$ IBER CHROMATIC DISPERSION has long been considered a main limitation in high bit rate and long-distance optical transmission systems. With the introduction of reconfigurable optical networking, any change in path lengths due to switching will produce a different accumulation of dispersion, thereby minimizing the effectiveness of fixed chromatic dispersion compensation solutions. Furthermore, future maintenance or scalability of an optical system may change the accumulated dispersion. Moreover, chromatic dispersion varies with temperature [1], becoming a significant problem for long-distance transmission of $\geq 40-\mathrm{Gb} / \mathrm{s}$ signals [2], [3]. All of these scenarios would necessitate tunable dispersion compensation and, therefore, the ability to monitor the actual data signal while the system is still online. For management and monitoring purposes in a robust system, a flexible and cost-effective dispersion monitoring technique could prove quite beneficial.

Of course, some level of dispersion monitoring can be achieved by actual electronic measurement of the eye-opening penalty, $Q$-factor, or bit-error rate (BER). However, these methods require electronics that are fairly difficult to implement for higher data rates. Previous reported work on optical techniques for dispersion monitoring has included: 1) measuring the phase delay between two out-of-band subcarrier

Manuscript received August 29, 2001; revised December 17, 2001.

M. N. Petersen is with the Research Center COM, Technical University of Denmark, 2800 Lyngby, Denmark (e-mail: mnp@com.dtu.dk).

Z. Pan and A. E. Willner are with the Department of Electrical Engineering-Systems, University of Southern California, Los Angeles, CA 90089-2565 USA.

S. Lee and S. A. Havstad are with Phaethon Communications, Fremont, CA 94538 USA.

Publisher Item Identifier S 1041-1135(02)01668-3. tones [4]; 2) adding a phase modulation at the transmitter and measuring the phase-to-amplitude modulation conversion at the receiver [5]; and 3) modulating the frequency of the transmitted data signal and then monitoring the clock phase deviation at the receiver [6].

We demonstrate a simple technique for dispersion monitoring by adding a single inband subcarrier tone to the transmitted data signal. A measurable dispersion of up to $1200 \mathrm{ps} / \mathrm{nm}$ is demonstrated in a 10-Gb/s channel using a $7-9 \mathrm{GHz}$ subcarrier and the addition of the subcarrier induced a power penalty of $<0.5 \mathrm{~dB}$. Dynamic ranges exceeding $20 \mathrm{~dB}$ and resolution sensitivities better than $10(\mathrm{ps} / \mathrm{nm}) / \mathrm{dB}$ are shown. $10(\mathrm{ps} / \mathrm{nm}) / \mathrm{dB}$ means that a $10 \mathrm{ps} / \mathrm{nm}$ dispersion change will give rise to $1-\mathrm{dB}$ power change in the electrical monitor signal for this particular system. With an $8-\mathrm{GHz}$ tone and a $15 \%$ modulation depth for $10-\mathrm{Gb} / \mathrm{s}$ signals, we show a measurement range of 975 $\mathrm{ps} / \mathrm{nm}$ with a 22-dB dynamic range. We use the monitor output signal to achieve accurate tunable dispersion compensation and demonstrate a reduction in power penalty from $2 \mathrm{~dB}$ to less than $0.5 \mathrm{~dB}$. In general, this technique has a reconfigurable measurement range.

\section{OPERATION PRINCIPLE}

In general, chromatic dispersion causes periodic power fading of an RF subcarrier tone after detection by a square law detector [7]. The reason is that the frequency-dependent dispersion produces a deleterious time delay between the transmitted sidebands, thereby causing serious RF power fading. This effect is problematic for subcarrier multiplexed systems. However, RF fading can be exploited for dispersion monitoring if a sinusoidal subcarrier signal is added at the transmitter as a tone in digital systems.

Fig. 1 shows the basic principle of RF fading of a subcarrier tone within the data band. The tone carries no data and has a narrow spectral linewidth that is less than a few megahertz. Since the tone is within the frequency band of the data, it experiences the same dispersion as the data and can be used as a dispersion sensor. The upper and lower side bands of the subcarrier will initially be inphase with each other at the transmitter. However, a phase difference will accumulate during transmission between the two sidebands as a result of the frequency-dependent fiber chromatic dispersion. The degree of phase mismatch is related to the amount of accumulated dispersion when the subcarrier is optoelectronically detected and the subcarrier power fades according to the phase mismatch in a predictable 




Fig.1. Principle of RF fading used for dispersion monitoring. RF tone within data band fades due to dispersion.

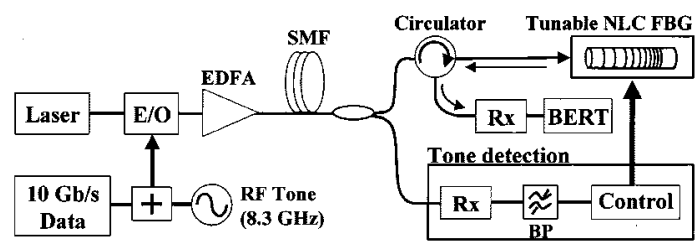

Fig. 2. Setup used for dispersion monitoring and compensation using inband tone as dispersion sensor.

manner. The detected photocurrent at the tone frequency will fade according to (1)

$$
I\left(f_{S C}\right)=I_{0} m \cos \left(\frac{\pi f_{S C}^{2} \lambda D L}{c}\right)
$$

where $I_{0}$ is the peak photocurrent, $m$ is the modulation depth, $f_{S C}$ is the subcarrier tone frequency, $\lambda$ is carrier wavelength, $D L$ is the total accumulated dispersion, and $c$ is the speed of light in vacuum.

The dispersion monitor resolution and measurement range are dependent on the specific frequency of the subcarrier tone. The tone frequency determines the point of total fading, for which unambiguous measurement requires the range of dispersion to be within one period of fading. Therefore, the point of total fading is referred to as the measurement the range. Consequently, by changing the tone frequency it is possible to select the measurable dispersion range and the sensitivity accordingly.

\section{EXPERIMENTAL SETUP}

Fig. 2 shows the experimental setup used for dispersion monitoring. The optical source is modulated by a combined signal consisting of $10-\mathrm{Gb} / \mathrm{s}$ data and a single frequency tone in the range of 7-9 GHz. The modulation depth of the subcarrier tone relative to the data is $\leq 15 \%$. The $\{$ data + subcarrier $\}$ modulated signal is transmitted through conventional singlemode fiber of different lengths to simulate different amounts of accumulated dispersion. Some optical power is tapped off before the data receiver in order to detect the subcarrier monitoring tone. This tone is extracted after detection by using a tunable electrical bandpass filter. Note that this technique uses a very simple setup and all equipment used for the dispersion monitoring system is commercially available without requiring any fast electronics.

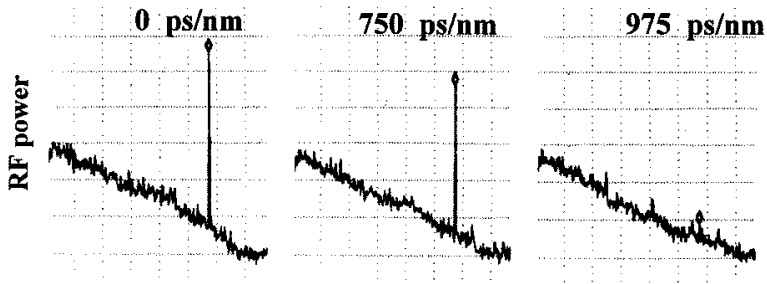

(a)

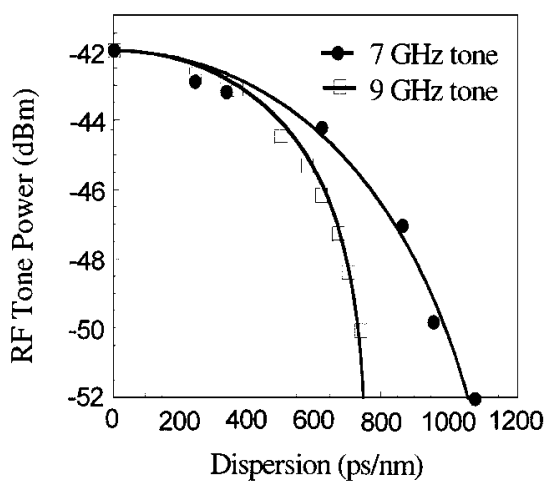

(b)

Fig. 3. (a) RF spectra of an $8-\mathrm{GHz}$ tone in a $10-\mathrm{Gb} / \mathrm{s}$ transmission system at different amounts of accumulated dispersion. (b) RF fading due to dispersion for a 7- and 9-GHz tone. The solid lines represent the theoretical results and the points are experimental results.

A nonlinearly chirped FBG, which has the periodicity that varies nonlinearly along the length of the fiber, is used as a tunable dispersion compensator [8]. This FBG produces a time delay that varies nonlinearly with wavelength. When it is stretched uniformly, the dispersion at a specific wavelength is changed. The FBG is controlled by the detected RF power to compensate for the accumulated dispersion.

\section{RESUlTS AND DISCUSSION}

In our experiment, we transmitted the optical signal through different lengths of fiber and measured the electrical subcarrier tone power for each transmission length; note that we maintain a constant optical power before the receiver. Fig. 3(a) shows the observed electrical power spectrum for different accumulated dispersions. The total fading of the $8-\mathrm{GHz}$ tone occurs at $975 \mathrm{ps} / \mathrm{nm}$. Fig. 3(b) shows the measured and theoretical fading curves for tone frequencies of 7 and $9 \mathrm{GHz}$, in which the fading follows a $\sin ^{2}$ function with increasing dispersion. With a 7- and 9-GHz subcarrier, a range of 675 and $1200 \mathrm{ps} / \mathrm{nm}$, respectively, is possible with sensitivities up to $10(\mathrm{ps} / \mathrm{nm})$ in according to $1 \mathrm{~dB}$ of power fading. Based on Fig. 3(b), an important characteristic of this technique is that the sensitivity increases for higher dispersion values since higher values reside on a steeper portion of the curve.

The subcarrier fading is also sensitive to differential group delay (DGD). However, it was experimentally verified that DGD values up to $10-15$ ps do not influence the dispersion measurement. At larger values additional fading will be observed.

range results in a higher sensitivity.It is possible to reconfigure the measurement range and sensitivity of the monitor by simply tuning the tone frequency. Fig. 4 shows the relationship between the tone frequency and the measurable dispersion range, in which a smaller Using the high subcarrier frequency 


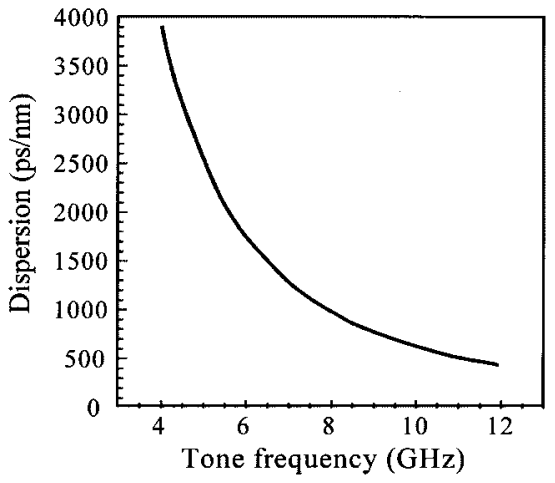

Fig. 4. Theoretical curve for measurable range of chromatic dispersion.

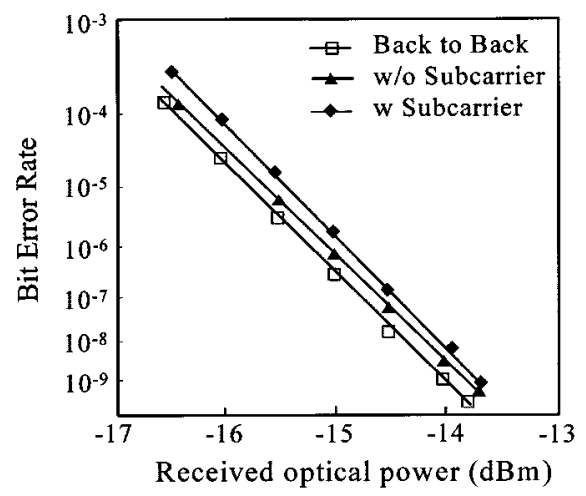

Fig. 5. Measured BERs. The inband tone is at $8.0 \mathrm{GHz}$ and at $15 \%$ modulation depth. The 3-dB bandwidth of the tone is $5 \mathrm{MHz}$.

will provide higher sensitivity. However in order not to occupy more bandwidth, the frequency cannot be out of the data band. On the other hand, using the low subcarrier frequency will provide a wider measurement range, but the sensitivity will not be enough for dispersion compensation. A 3-GHz subcarrier will for example enable a measurement range of about $7000 \mathrm{ps} / \mathrm{nm}$, but a 40-km transmission in single-mode fiber will cause less than $0.5-\mathrm{dB}$ drop in the $3-\mathrm{GHz}$ subcarrier power.

We evaluated the system impairment caused by the addition of the subcarrier tone to the data at the transmitter. The higher the modulation index of the subcarrier is, the higher the system impairment will be. Fig. 5 shows the BER measurements for the back-to-back and the 30-km transmission cases, with and without the $15 \%$ modulation depth $8-\mathrm{GHz}$ subcarrier. A $<0.5-\mathrm{dB}$ power penalty is observed due to the inclusion of the monitor tone and complete tone fading occurs at $975 \mathrm{ps} / \mathrm{nm}$. Measurements for several tone frequencies in the range of 7 to $9 \mathrm{GHz}$ found no measurable difference in power penalty.

By using the measured tone power as a control signal, we optimize the system by stretching the nonlinearly chirped FBG that has a dispersion range of -350 to $-1000 \mathrm{ps} / \mathrm{nm}$. We transmit the signal through $60 \mathrm{~km}$ of fiber and accumulate $830 \mathrm{ps} / \mathrm{nm}$ of dispersion. We use an $8.3-\mathrm{GHz}$ tone corresponding to a measurement range of $910 \mathrm{ps} / \mathrm{nm}$. Fig. 6(a) shows the observed eye diagrams and electrically filtered tone before and after compensation. In this experiment, a $22-\mathrm{dB}$ rise in tone power is achieved due to dispersion compensation. The BER measurement results

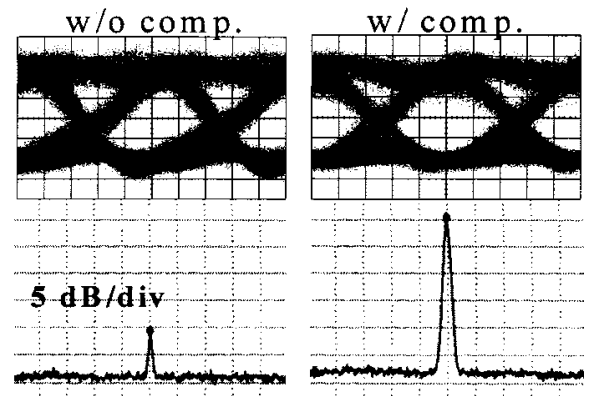

(a)

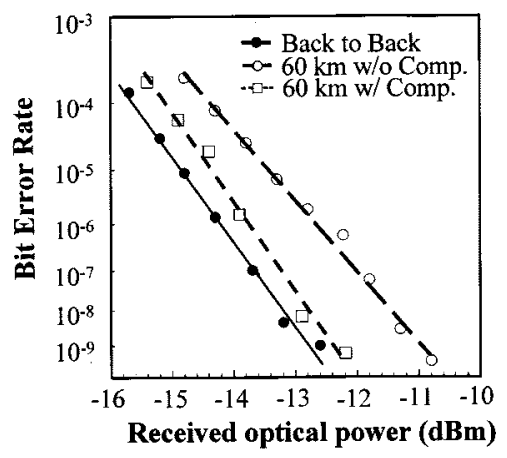

(b)

Fig. 6. (a) Eye of data and filtered tone before and after dispersion compensation. A 22-dB rise in tone power is observed. (b) BER curves showing reduction of power penalty due to dispersion compensation.

are shown in Fig. 6(b). The power penalty is reduced from $2 \mathrm{~dB}$ to less than $0.5 \mathrm{~dB}$ due to the dispersion compensation.

In summary, we demonstrate a simple technique for dispersion monitoring by adding a single inband tone to the transmitted data. We also show tunable dispersion compensation by using the measured tone power as the control signal to vary a nonlinearly chirped FBG.

\section{REFERENCES}

[1] W. H. Hatton and M. Nishimura, "Temperature dependence of chromatic dispersion in single mode fibers," J. Lightwave Technol., vol. LT-4, pp. $1552-1555,1986$.

[2] S. Kuwahara, A. Sano, K. Yonenaga, Y. Miyamoto, and Y. Yamabayashi, "Adaptive dispersion equalization by detecting dispersion fluctuations using PM-AM conversion," Electron. Lett., vol. 34, no. 20, pp. 1956-1958, 1998.

[3] A. Sano, T. Kataoka, M. Tomizawa, K. Hagimoto, K. Sato, K. Wakita, and K. Kato, "Automatic dispersion equalization by monitoring extracted-clock power in a 40-Gbit/s, 200-km transmission line," in ECOC, 1996, TuD.3.5, pp. 2207-2210.

[4] T. E. Dimmick, G. Rossi, and D. J. Blumenthal, "Optical dispersion monitoring technique using double sideband subcarriers," IEEE Photon. Technol. Lett., vol. 12, pp. 900-902, July 2000.

[5] M. Tomizawa, Y. Yamabayashi, Y. Sato, and T. Kataoka, "Nonlinear influence on PM-AM conversion measurement of group velocity dispersion in optical fibers," Electron. Lett., vol. 30, no. 1434, 1994.

[6] Y. Takushima and K. Kikuchi, "In-service monitor for group-velocity dispersion of optical fiber transmission systems," Electron. Lett., vol. 37, pp. 743-745, 2001.

[7] F. Devaux, Y. Sorel, and F. Kerdiles, "Simple measurement of fiber dispersion and of chirp parameter of intensity modulated light emitter," $J$. Lightwave Technol., vol. 11, pp. 1937-1940, Dec. 1993.

[8] K.-M. Feng, J.-X. Cai, V. Grubsky, D. S. Starodubov, M. I. Hayee, S. Lee, X. Jiang, A. E. Willner, and J. Feinberg, "Dynamic dispersion compensation in a 10-Gb/s optical system using novel voltage tuned nonlinearly-chirped fiber bragg grating," IEEE Photon. Technol. Lett., vol. 11, pp. 373-375, Dec. 1999. 